\title{
Ultraestrutura comparativa da língua do sagui-de-tufo-preto (Callithrix penicillata) e do bugio-preto (Alouatta caraya) em diferentes faixas etárias ${ }^{1}$
}

\author{
Cayo Y. Nitta ${ }^{2 *}$, Luana C.S. Silva ${ }^{3}$, Maria A. Miglino ${ }^{3}$, Carlos E. Ambrosio ${ }^{4}$, \\ Pedro P. Bombonato ${ }^{3}$ e Rose E.G. Rici ${ }^{3}$
}

\begin{abstract}
Nitta C.Y., Silva L.C.S., Miglino M.A., Ambrosio C.E., Bombonato P.P. \& Rici R.E.G. 2013. [Comparative ultrastructure of the tongue of black-tufted-ear-marmoset (Callithrix penicillata) and black-howler-monkey (Alouatta caraya) in different age groups.] Ultraestrutura comparativa da língua do sagui-de-tufo-preto (Callithrix penicillata) e do bugio-preto (Alouatta caraya) em diferentes faixas etárias. Pesquisa Veterinária Brasileira 33(Supl.1):75-84. Faculdade de Medicina Veterinária e Zootecnia, Universidade de São Paulo, Av. Prof. Orlando Marques de Paiva 87, Cidade Universitária, São Paulo, SP 05508-270, Brazil. E-mail: cayo.nitta@usp.br

For the comparative analysis and description of the morphology of the tongue and taste buds as well as the distribution of these we have used three animals of different age groups (puppy, young adult), of two species of primate, Callithrix penicillata (black-tufted-ear-marmoset) and Alouatta caraya (black-howler-monkey), both being observed by scanning electron microscopy. The animals of the species Callithrix penicillata were from a commercial breeding of wild animals in Atibaia-SP and they had died of natural causes, and the specie Alouatta caraya were from the collection of Macroscopic Anatomy Laboratory of Faculty of Veterinary Medicine and Animal Science University of São Paulo (FMVZ-USP). With the material analyzed and the technique employed could be seen that the tongue of black-tufted-ear-marmosets and black-howler-monkeys had examined lingual papillae similar to those described for other primate species. The tongue has morphological differences, especially for some types of lingual papillae, at the expense of advancing age of the animals. Overall, we found four types of lingual papillae, which are: filiform, fungiform, vallate and litter. The tongues of the black-howler-monkey had characteristics of animals with herbivorous diets. Possessing this way with the filiform papillae varied formats (crown, spear, multifilament), according to the regions of the tongue, and also robust taste, especially in the lingual prominence. Since the tongues of the black-tufted-ear-marmosets owned features of omnivorous animals. With filiform-shaped crown, large amounts of fungiform papillae and foliate papillae developed. It was observed that the changes of tongue papillae that occur in animals after birth correlated with the change in the types of foods consumed. Being suspected then conclude that diets have direct relation to the morphological and structural of the lingual papillae now here in the animals analyzed.
\end{abstract}

INDEX TERMS: Lingual papillae, scanning electron microscopy, Callithrix penicillata, Alouatta caraya.

\footnotetext{
${ }^{1}$ Recebido em 21 de outubro de 2013.

Aceito para publicação em 29 de novembro de 2013.

${ }^{2}$ Discente de Medicina Veterinária, Faculdade de Medicina Veterinária e Zootecnia (FMVZ), Universidade de São Paulo (USP), Av. Prof. Orlando Marques de Paiva 87, Cidade Universitária, São Paulo, SP 05508-270, Brasil. *Autor para correspondência: cayo.nitta@usp.br

${ }^{3}$ Departamento de Cirurgia, FMVZ-USP, São Paulo, SP.

${ }^{4}$ Departamento de Medicina Veterinária, Faculdade de Zootecnia e Engenharia de Alimentos- (FZEA), USP, Rua Duque de Caxias Norte 225, Pirassununga, SP13635-900, Brasil.
}

RESUMO.- Para a análise e descrição comparativa da morfologia da língua e de suas papilas, bem como a distribuição destas, foram utilizados três animais de faixas etárias distintas (filhote, jovem, adulto), de duas espécies de primatas, Callithrix penicillata (sagui-de-tufo-preto) e Alouatta caraya (bugio-preto), ambas sendo observadas através da microscopia eletrônica de varredura. Os animais da espécie Callithrix penicillata eram procedentes de um criadouro comercial de animais selvagens em Atibaia/SP e vieram a 
óbito por causas naturais, e os da espécie Alouatta caraya eram provenientes do acervo do Laboratório de Anatomia Macroscópica da Faculdade de Medicina Veterinária e Zootecnia da Universidade de São Paulo (FMVZ-USP). Com o material analisado e a técnica empregada pôde-se constatar que as línguas dos saguis-de-tufo-preto e dos bugios-pretos analisadas apresentavam papilas linguais similares às descritas para outras espécies de primatas. As línguas possuíam diferenças morfológicas, principalmente para alguns tipos de papilas linguais, em detrimento do avançar da idade dos animais. De forma geral, foram observados quatro tipos de papilas linguais, sendo estas: filiforme, fungiforme, valada e folhada. As línguas dos bugio-pretos apresentavam características de animais com dietas herbívoras. Possuindo desta forma papilas filiformes com formatos variados (coroa, lança, multifilamentar), de acordo com as regiões da língua, e também robustas papilas, principalmente na proeminência lingual. Já as línguas dos saguis-de-tufo-preto possuíam características de animais onívoros. Com papilas filiformes em formato de coroa, grandes quantidades de papilas fungiformes e desenvolvidas papilas folhadas. Observou-se que as modificações das papilas linguais que ocorrem nos animais após o nascimento apresentaram correlação com a mudança nos tipos de alimentos consumidos. Sendo presumível então concluir que as dietas possuem relação direta para com as alterações morfológicas e estruturais das papilas linguais nos animais ora aqui analisados.

TERMOS DE INDEXAÇÃO: Papilas linguais, microscopia eletrônica de varredura, Callithrix penicillata, Alouatta caraya.

\section{INTRODUÇÃO}

Callithrix penicillata (Geoffroy, 1812) assim como outros saguis do gênero Callithrix, são classificados como primatas do novo mundo, apresentado uma pelagem estriada no dorso e cauda, e com ornamentos pilosos nas orelhas. A presença de tufos pré-auriculares longos e negros, em forma de pincel assegura-lhe o nome de sagui-de-tufo-preto e os diferenciam de outras espécies do gênero (Auricchio 1995).

Esses pequenos primatas habitam a Floresta Amazônica, a Mata Atlântica e principalmente o Cerrado brasileiro, formando grupos de, em média, 10 indivíduos. Apresentam uma massa corpórea em media de 350 gramas, vivem cerca de 10 anos em vida livre e 16 anos em cativeiro e atingem a maturidade sexual aos 18 meses. As fêmeas apresentam uma gestação de aproximadamente 140 dias e os filhotes nascem com 20-35g, sendo amamentados por cerca de 100 dias. Já com duas semanas os filhotes começam a experimentar algumas frutas maduras, mas sem deixar a amamentação que finda aos dois meses de idade (Faria 1989; Auricchio 1995). São considerados insetívoros-gumívoros, alimentando-se de grande variedade de matéria vegetal, como os exsudatos de árvores, sementes, flores, frutos e néctar e insetos (Auricchio 1995, Castro 2003).

Alouatta caraya (Humboldt, 1812) é conhecido popularmente como bugio-preto ou macaco-roncador, devido a sua grande capacidade de vocalização em detrimento às modificações anatômicas do osso hióide. Apresentam médias de massa corpórea de 6-7 kg, altura de $30-75 \mathrm{~cm}$ e cauda de $80 \mathrm{~cm}$. Possuem uma longevidade de aproximadamente 20anos. Sua maturidade sexual é atingida com 24-40 meses de idade e o período de gestação é de cerca de 180 dias com desmame dos filhotes aos seis meses (Nowak 1999).

São encontrados em florestas de galeria e florestas semideciduais e abarcam como dieta alimentar a ingestão de folhas, seguida de frutas e flores (Santini 1985, Aurichio 1995).

A destruição do habitat, caça predatória, tráfico de animais silvestres e a manutenção de tais primatas como animais de estimação constituem atualmente as maiores ameaças à vida desses animais (Castro 2003) Assim o interesse pela pesquisa, conservação e recuperação dos mesmos começa a ganhar importância na sociedade atual, por conseguinte são necessários estudos mais detalhados sobre a estrutura dos mais diferentes sistemas nesses animais. Em particular, os primatas por apresentarem uma proximidade filogenética com os seres humanos, são objetos de estudo para avaliações nas mais diversas áreas, tais como parâmetros fisiológicos, nutrição, farmacologia, toxicologia, dentre outros (Clark 1994).

A língua é um órgão pertencente ao aparelho digestório que detém variadas funções, como captura e manipulação dos alimentos, modulação vocal, resfriamento corporal em alguns animais e atuando também como órgão sensorial de degustação (König \& Liebich 2004). As papilas linguais presentes contribuem para algumas dessas funções, e devido a isto, têm sido foco de estudos nos mais variados animais domésticos e silvestres. A distribuição de tais papilas na superfície da língua é característica de cada gênero animal e pode até distinguir uma espécie de outra. Sendo que um dos elementos que mais contribuem para as diferenças na morfologia, distribuição e no tipo destas papilas é a dieta que o animal ingere (Pastor et al. 2008).

Este estudo tem por finalidade descrever de forma comparativa a morfologia da língua e a distribuição de suas papilas nas espécies de primatas Callithrix penicillata e Alouatta caraya em diferentes idades através da microscopia eletrônica de varredura. As funções atribuídas às estruturas presentes na língua podem ser influenciadas pelo tipo de dieta do animal o que irá tornar a mucosa, as papilas linguais e os receptores gustatórios especializados.

\section{MATERIAL E MÉTODOS}

Para esta pesquisa foram utilizadas três línguas de saguis-de-tufo-preto (Callithrix penicillata) e três línguas de bugios-pretos (Alouatta caraya). A idade aproximada dos animais foi estimada com base no fenótipo e nos dados fornecidos pelas instituições de origem; assim, os animais foram classificados em: filhote, jovem e adulto. As amostras da espécie Callithrix penicillata eram compostas por línguas de um filhote (2 dias), uma jovem (3 anos) e uma adulta ( 9 anos). Já as amostras de Alouatta caraya analisadas eram constituídas de línguas de um filhote ( 6 dias), uma jovem ( 5 anos) e uma adulta (15 anos). Todos os animais analisados vieram a óbito por causas naturais. Os saguis-de-tufo-preto eram provenientes de um criadouro comercial legalizado de animais selvagens localizado na cidade de Atibaia/SP (Brasil) e foram doados ao Programa de Pós-Graduação de Anatomia dos Animais Domésticos e Silvestres da Faculdade de Medicina Veterinária e 
Zootecnia da Universidade de São Paulo (FMVZ-USP) para estudos anatômicos. E os bugio-pretos eram provenientes do acervo do Laboratório de Anatomia Macroscópica da FMVZ-USP onde anteriormente já haviam sido utilizados em outros estudos anatômicos. Tais animais estavam sendo mantidos e fixados em solução de formol $10 \%$.

Para a remoção das línguas da cavidade oral em todos os primatas analisados foi efetuado uma incisão na linha mediana ventral da região mentoniana e transversalmente ao logo de todo o frênulo lingual, para que em seguida as mesmas pudessem ser mensuradas através do uso de um paquímetro com precisão de $0,01 \mathrm{~cm}$. Posteriormente as amostras foram lavadas com solução PBS 0,10\% (Tampão fosfato-salino), pós-fixadas em solução de tetróxido de Ósmio a 1\% em PBS 0,1\% por 2 horas, lavadas por 45 minutos com solução PBS 0,10\%, e por 20 minutos em água destilada. Ao final o material foi desidratado em séries crescentes de alcoóis em concentrações de 50\%, 70\%, 90\% e 100\%, durante 30 minutos em cada série. Sendo então secas em aparelho de ponto crítico Balzers CPD 020 (FMVZ-USP) com o uso de $\mathrm{CO}_{2}$ líquido. Após tal procedimento os espécimes foram montados em bases metálicas de alumínio (stub) apropriadas para a microscopia eletrônica de varredura, utilizando-se cola de carbono. Na seqüência, foram submetidas a um revestimento metálico ("sputting") com ouro no aparelho metalizador EMITECH K550 (FMVZ-USP), sendo analisadas e fotografadas em microscópio eletrônico de varredura LEO 435VP (FMVZ-USP).

\section{RESULTADOS}

As línguas dos saguis-de-tufo-preto jovem e adulta continham uma raiz larga e espessa, um corpo alongado e um ápice arredondado, todavia no filhote a língua se apresentou com formato de colher. Não foram observados tórus lingual nem sulco mediano em nenhuma das línguas analisadas. Dados da mensuração individual de cada língua constam no Quadro 1.

As línguas dos bugios-Pretos fêmeas (Alouatta caraya) não apresentaram diferença na morfologia quando comparados à idade, visto que apenas os tamanhos destas variaram. De forma geral, apresentaram um ápice arredondado, um corpo alongado e uma base larga, caracterizando um formato de espátula. Nesta superfície dorsal foi observada uma proeminência na mucosa, no entanto nenhum sulco mediano foi verificado. Dados da mensuração individual das amostras da espécie Alouatta caraya constam no Quadro 2.

Quadro 1. Análises morfométricas das línguas de três espécimes de Callithrix penicillata com diferentes faixas etária

\begin{tabular}{lcccc}
\hline Amostras & Comprimento & $\begin{array}{c}\text { Largura } \\
\text { do ápice }\end{array}$ & $\begin{array}{c}\text { Largura } \\
\text { do corpo }\end{array}$ & $\begin{array}{c}\text { Largura } \\
\text { da raiz }\end{array}$ \\
\hline Filhote & $1,2 \mathrm{~cm}$ & $0,4 \mathrm{~cm}$ & $0,6 \mathrm{~cm}$ & $0,7 \mathrm{~cm}$ \\
Jovem & $2,4 \mathrm{~cm}$ & $0,6 \mathrm{~cm}$ & $0,8 \mathrm{~cm}$ & $1,0 \mathrm{~cm}$ \\
Adulto & $2,6 \mathrm{~cm}$ & $0,6 \mathrm{~cm}$ & $0,9 \mathrm{~cm}$ & $1,2 \mathrm{~cm}$
\end{tabular}

Quadro 2. Análises morfométricas das línguas de três espécimes de Alouatta caraya com diferentes faixas etárias

\begin{tabular}{lcccc}
\hline Amostras & Comprimento & $\begin{array}{c}\text { Largura } \\
\text { do ápice }\end{array}$ & $\begin{array}{c}\text { Largura } \\
\text { do corpo }\end{array}$ & $\begin{array}{c}\text { Largura } \\
\text { da raiz }\end{array}$ \\
\hline Filhote & $3,4 \mathrm{~cm}$ & $1,2 \mathrm{~cm}$ & $1,5 \mathrm{~cm}$ & $1,6 \mathrm{~cm}$ \\
Jovem & $4,8 \mathrm{~cm}$ & $1,5 \mathrm{~cm}$ & $1,8 \mathrm{~cm}$ & $2,0 \mathrm{~cm}$ \\
Adulto & $5,8 \mathrm{~cm}$ & $1,8 \mathrm{~cm}$ & $2,0 \mathrm{~cm}$ & $2,2 \mathrm{~cm}$
\end{tabular}

Nas duas espécies de primatas estudadas foi possível observar que na superfície ventral das amostras havia uma mucosa lisa com ausência de qualquer tipo de papila lingual. No entanto, a superfície dorsal apresentou uma mucosa com quatro tipos diferentes de papilas linguais, sendo estas: folhada, fungiforme, valada e filiforme.

Nos saguis-de-tufo-preto as papilas filiformes estavam distribuídas irregularmente ao longo da superfície dorsal das três línguas. Com maior concentração e desenvolvimento no corpo e ápice da língua. Também foram identificadas papilas filiformes simples e ramificadas em todas as amostras. No entanto, estas características foram distintas conforme as idades dos animais analisados.

Nos bugios-pretos as papilas filiformes estavam distribuídas ao longo da superfície dorsal, predominantemente no corpo e ápice da língua. Na fêmea jovem e adulta foram identificadas papilas filiformes com formatos distintos, de acordo com regiões específicas da língua. E assim como foi observado com outras papilas linguais, as papilas filiformes nesta espécie animal eram diferentes em conformidade com o avançar da idade.

Com relação ao filhote de sagui-de-tudo-preto observaram-se na superfície da mucosa dorsal da língua as papilas filiformes, fungiformes, valadas e folhadas (Fig.1A). As papilas filiformes foram as mais prevalentes e estavam distribuídas no ápice e no corpo da língua. No ápice, tais papilas eram menos desenvolvidas do que as demais presentes no corpo. As papilas não tinham estrutura definida, sendo encontradas do tipo simples, bifurcada e trifurcada (Fig.1B). Em geral, as orientações destas estruturas eram aleatórias. Já as papilas fungiformes estavam presentes em toda superfície dorsal, com exceção da base da língua. Estruturalmente eram maiores, mas em menor quantidade do que as primeiras. Macroscopicamente as papilas fungiformes possuíam um corpo cilíndrico da base ao seu ápice, não se assemelhando ao formato de um cogumelo, apresentando poros gustativos e sendo rodeadas por papilas filiformes. Ao todo três papilas valadas foram observadas na região caudal da língua, sendo uma papila medial e duas laterais, formando assim uma estrutura em " $V$ " invertido (Fig.1A). Macroscopicamente tais papilas apresentavam o corpo rodeado por sulcos profundos e uma superfície plana, com poucos poros gustativos. E por final na região dorso-lateral da base da língua observaram-se ainda as papilas folhadas com quatro folhas pouco desenvolvidas e também muitas aberturas de ductos da glândula salivar sublingual.

Já a despeito do animal jovem de Callithrix penicillata constatou-se a presença bem definida dos quatro tipos de papilas linguais já descritas anteriormente. Em que as papilas filiformes foram as mais numerosas e se encontravam distribuídas em toda a superfície dorsal, com exceção da base da língua. A estrutura variava de acordo com as regiões da língua e se orientavam no sentido caudal. No ápice e corpo da língua, inclusive nas margens, estas papilas apresentavam uma estrutura ramificada, em formato de coroa com uma área basal central côncava (Fig.1C). Nessas regiões, as papilas filiformes apresentaram de cinco a nove ramificações pontiagudas, que diminuíam conforme se avançava em sentido caudal. As as papilas fungiformes 


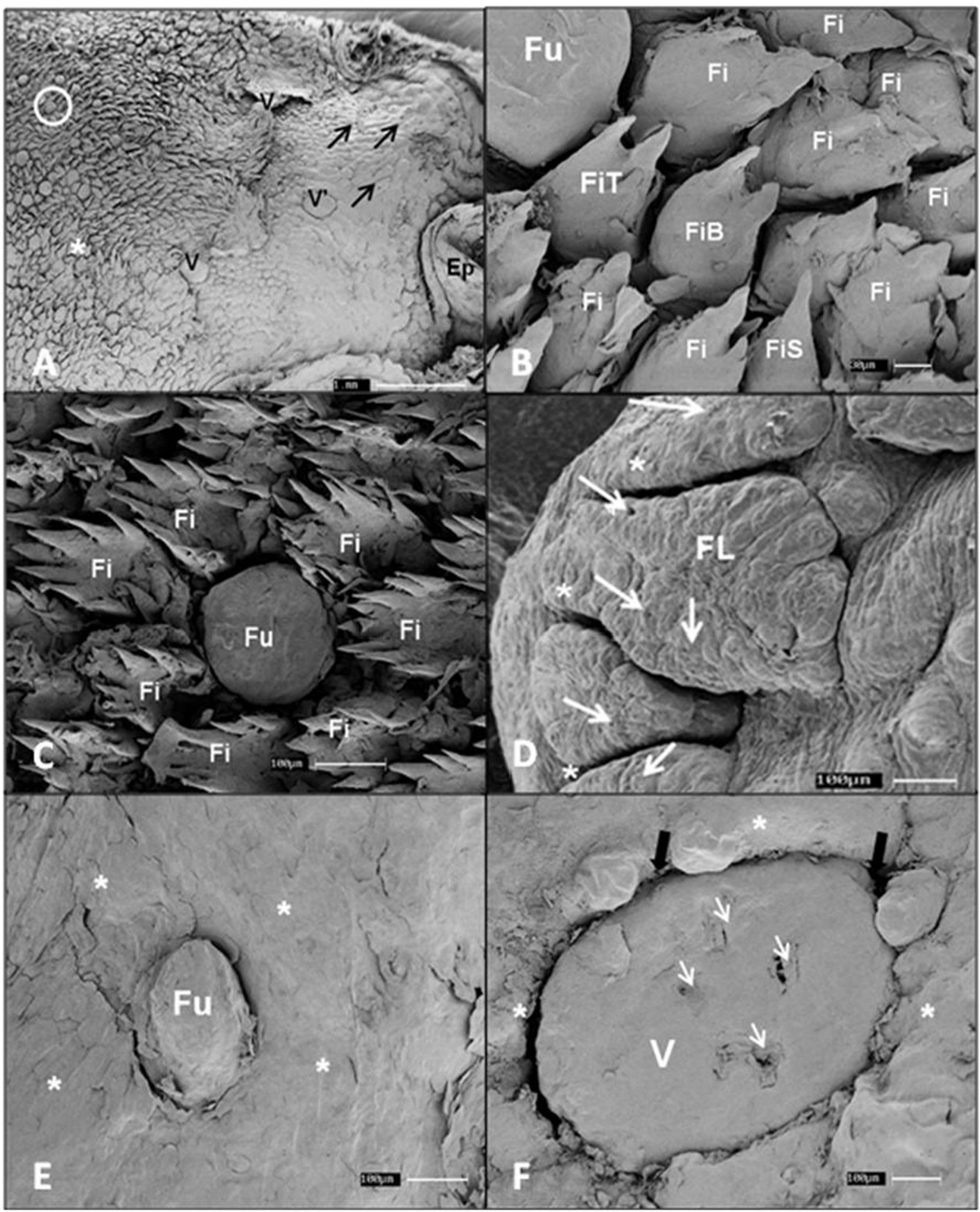

Fig.1. Eletromicrografias de varredura da língua de três espécimes de Callithrix penicillata com diferentes faixas etárias. (A) Animal filhote - Região caudal da língua. Presença de duas papilas valadas laterais (V) e uma papila valada medial (V') dispostas em um "V" invertido. Nesta região observa-se papilas fungiformes (círculo branco), papilas filiformes (asterisco branco) e papilas folhadas (asterisco preto). Nota-se a abertura de ductos da glândula salivar sublingual (setas pretas) próximas à epiglote (Ep). (B) Animal filhote - Região do ápice da língua. Detalhe em maior aumento de uma papila fungiforme (Fu) e papilas filiformes (Fi). Nota-se uma papila filiforme simples (FiS), bifurcada (FiB) e uma trifurcada (FiT). (C) Animal jovem - Presença de uma papila fungiforme (Fu) rodeada por papilas filiformes (Fi). (D) Animal jovem - Detalhe de uma papila folhada (FL) com suas dobras laterais $\left({ }^{*}\right)$. Nota-se a presença de poros gustativos em sua superfície (setas brancas). (E) Animal adulto - Detalhe da ausência de papilas filiformes (*) ao redor de uma papila fungiforme (Fu) na região do ápice da língua. (F) Animal adulto - Região da base da língua. Detalhe dos poros gustativos (setas brancas) na superfície de uma papila valada lateral (V), nota-se ao seu redor um sulco profundo (setas pretas) e a ausência de papilas filiformes $(*)$. 
foram encontradas nos dois terços craniais (corpo e ápice), estando mais presentes na região do corpo e na extremidade cranial do ápice da língua e com uma menor concentração no ápice, rodeadas pelas papilas filiformes (Fig.1C), apresentavam uma estrutura arredondada e ligeiramente convexa, semelhante a um "cogumelo" (Fig.1C) e em sua superfície achatada foram encontrados poros gustativos. $\mathrm{Na}$ região caudal da língua foram observadas grandes quantidades de forames dos ductos das glândulas salivares e papilas valadas dispostas em "V" invertido, sendo o ápice do "V" oposto a base lingual. Três papilas valadas observadas (uma medial e duas laterais) se apresentavam de forma arredonda estando margeadas por sulcos profundos e com poros gustativos em sua superfície. As papilas valadas laterais situadas no corpo da língua estavam cercadas pelas papilas filiformes, já a papila medial presente na base da língua, estava rodeada por prolongamentos da mucosa com formato de dedos. Na porção caudal da base da língua, especificamente na região dorso-lateral direita e esquerda, foram observadas papilas folhadas, caracterizadas pela disposição paralela de pequenas pregas laterais da mucosa lingual em forma de folhas, apresentavam-se com três dobras (folhas) com observação de poros gustativos em sua superfície (Fig.1D).

No sagui-de-tufo-preto adulto analisado constatou-se a presença de uma superfície dorsal ligeiramente lisa com papilas fungiformes, valadas e folhadas, não sendo observadas desta forma as papilas filiformes. As papilas fungiformes foram as mais predominantes estando distribuídas no ápice e principalmente no corpo da língua, mas ausentes na extremidade cranial do ápice da língua. Na porção mediana da língua as papilas fungiformes estavam distribuídas aleatoriamente e em maiores quantidades. Macroscopicamente tais papilas se apresentaram de forma rasa e sem o formato típico de cogumelo. Possuíam uma superfície desgastada com a existência de poucos poros gustativos, não estando rodeadas por papilas filiformes (Fig.1E). Na região caudal da língua foram observadas três papilas valadas, sendo uma papila medial próxima à faringe e duas laterais oposta à epiglote, no qual formavam uma estrutura semelhante a um V invertido. Macroscopicamente eram ovaladas e estavam rodeadas por um sulco profundo (Fig.1D). Sendo encontrados em sua superfície alguns poros gustativos (Fig.1F). Nas bordas laterais desta região, foram encontradas papilas folheadas com três dobras bem definidas e poucas aberturas da glândula salivar foram observadas.

Para o bugio-preto filhote observou-se a presença predominante das papilas filiformes no ápice e no corpo da língua, as quais de forma geral se orientavam no sentido caudal (Fig.2A). Não foram encontrados formatos definidos para tais papilas, no entanto as observadas no corpo foram maiores do que as presentes no ápice da língua. As papilas fungiformes estavam distribuídas aleatoriamente nos dois terços iniciais da língua (ápice e corpo), tendo um predomínio na ponta do ápice. Macroscopicamente nestas papilas foi possível observar poros gustativos e as mesmas possuíam um formato de "cogumelo" estando rodeadas pelas papilas filiformes. Na região da base da língua foram observadas três papilas valadas, sendo duas laterais e uma medial mais caudalmente, formando um "V" invertido (Fig.2B). Macroscopicamente eram as maiores papilas linguais observadas, apresentando uma estrutura larga e elíptica, circundadas por um sulco profundo. As papilas valadas laterais, diferentemente da papila valada medial, estavam rodeadas por papilas filiformes. Lateralmente à esta região foram encontradas duas papilas folhadas, sendo uma direita e a outra esquerda.

Quanto ao bugio-preto jovem analisado foi possível observar que as papilas filiformes eram as mais numerosas e estavam distribuídas ao longo do ápice e corpo da língua. Tais papilas se apresentavam sob três formatos distintos: coroa, lança e multifilamentar, em conformidade com determinadas regiões da língua. No ápice estas papilas possuíam um formato de coroa, no corpo à exceção da proeminência lingual apresentavam o formato de lança e na proeminência lingual eram do tipo multifilamentar. As papilas fungiformes foram observadas na região do ápice e corpo lingual, apresentando uma estrutura com formato de "cogumelo" rodeado por papilas filiformes (Fig.2C). Em suas superfícies notou-se a presença de poros gustativos (Fig.2D) e na região do corpo três papilas fungiformes, alinhadas lateralmente à proeminência lingual, tanto do lado esquerdo quanto direito. Três papilas valadas situadas na região da base da língua estavam dispostas de forma triangular. Macroscopicamente tais papilas possuíam o corpo rodeado por sulcos profundos e uma superfície plana, no qual não foram observados poros gustativos. Lateralmente nesta região observaram-se as papilas folhadas direita e esquerda.

Em Alouatta caraya adulto foi observado que a superfície da sua língua estudada apresentava um aspecto aveludado, em detrimento da grande quantidade de papilas filiformes distribuídas na região de ápice e corpo da língua. Tais papilas apresentaram variações quanto às suas morfologias, tendo no ápice um formato de coroa, no corpo um formato de lança (Fig. 2E) e já na proeminência lingual um formato multifilamentoso. Nos dois terços anteriores (corpo e ápice) da língua, foram observadas papilas fungiformes com formato de "cogumelos", estando estas rodeadas por papilas filiformes (Fig.2E). Em suas superfícies foram encontrados poros gustativos. Na região do corpo lingual, lateralmente à proeminência, observou-se um agrupamento (direito e esquerdo) de três papilas fungiformes. Na região da base da língua, próximo à epiglote, foram encontradas três papilas valadas, sem a presença de poros gustativos. Sendo duas papilas valadas laterais, envolvidas por papilas filiformes, e uma papila medial, envolvidas parcialmente em algumas papilas filiformes. Nesta região foram também encontradas aberturas de ductos de glândulas salivares (Fig.2F). Ainda na base da língua, na região dorso-lateral direita e esquerda, foram observadas as papilas folhadas, caracterizadas pela disposição paralela de suas pregas em forma de folhas. Não foram observados poros gustativos evidentes em tais papilas.

\section{DISCUSSÃO}

A morfologia da língua bem como a presença, distribuição e estrutura das papilas linguais estão correlacionadas com os hábitos alimentares do animal em determinado hábitat, 


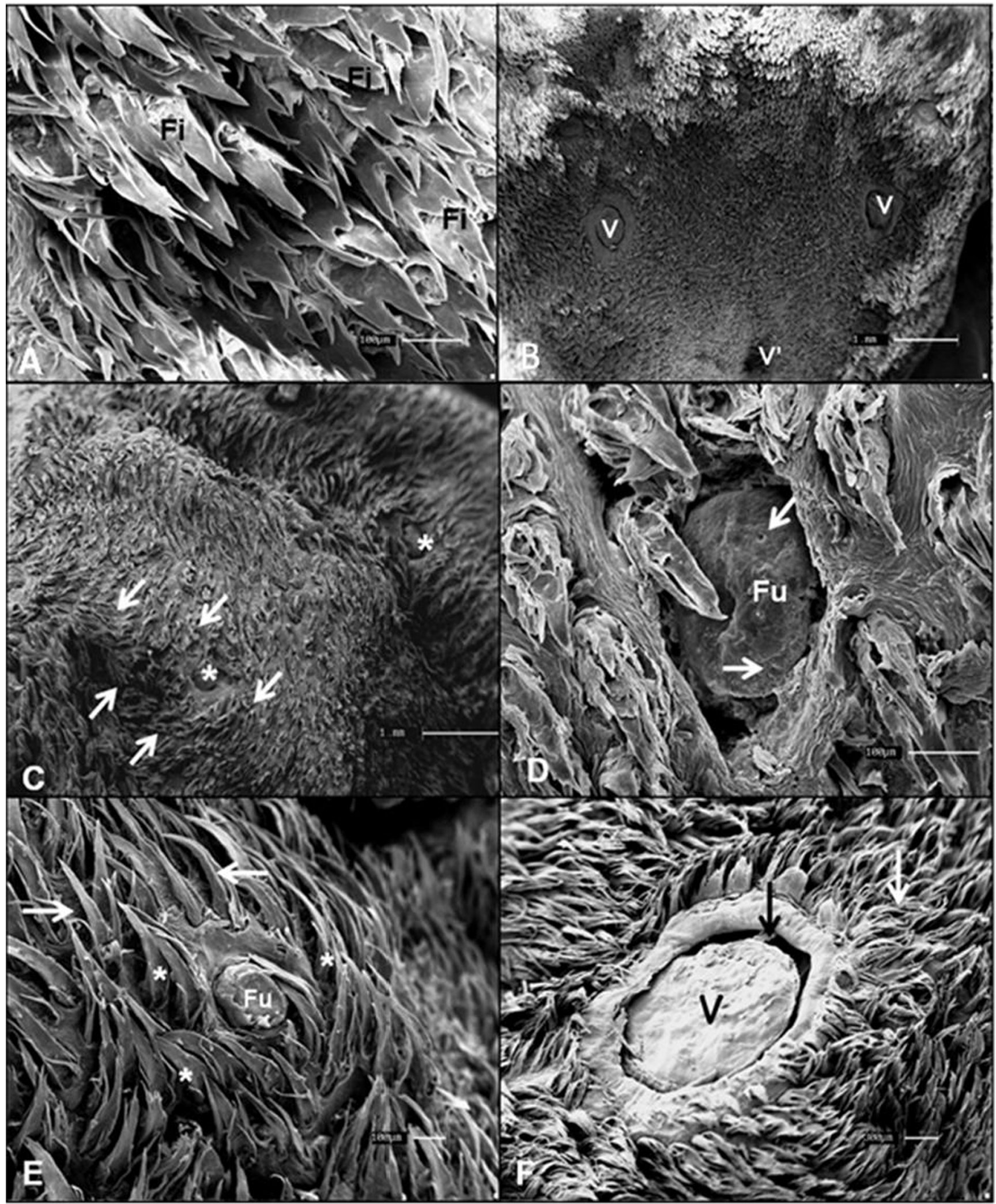

Fig.2. Eletromicrografias de varredura da língua de três espécimes de Alouatta caraya com diferentes faixas etárias. (A) Animal filhote - Detalhe das papilas filiformes com formato de lança, presentes no corpo da língua. (B) Animal filhote - Região caudal da língua. Evidencia-se duas papilas valadas laterais (V) e uma papila valada medial (V'), dispostas em V invertido. (C) Animal jovem - Evidencia-se papilas fungiformes $\left(^{*}\right.$ ) rodeadas por papilas filiformes (setas brancas). (D) Animal jovem - Corpo da língua. Detalhe de poros gustativos (setas brancas) na superfície de uma papila fungiforme (Fu). (E) Animal adulto - Corpo da língua. Detalhe de uma papila fungiforme $(\mathrm{Fu})$ rodeada por papilas filiformes $\left({ }^{*}\right)$ que apresentam formato de lança (setas brancas). (F) Animal adulto - Região caudal da língua. Observa-se uma papila valada lateral (V) cercada por um sulco profundo (seta preta) e papilas filiformes (seta branca). 
podendo assim sofrer variações e adaptações (Jackowiak et al. 2005, Pastor et al. 2008).

Segundo Doran \& Baggett (1971) as línguas dos mamíferos podem ser divididas em duas categorias funcionais, cada uma com clara correlação morfológica. 0 tipo I, encontrados em alguns primatas, possui formato de "espátula" e apresenta principalmente uma função intra-oral durante a mastigação. Já o tipo II, com formato "fusifome" é caracterizada principalmente pela função extra-oral na recolha de alimentos, como ocorrem em alguns monotremados. No presente estudo, as línguas analisadas de Callithrix penicillata apresentaram uma morfologia compatível com o tipo I, ou seja, um corpo alongado e um ápice arredondado, sendo assim, compatível com a sua dieta.

Em nosso estudo, não foram observados sulcos medianos na superfície dorsal nas línguas analisadas das duas espécies de primatas, diferentemente como foi relatado para alguns primatas por Sonntag (1921), Ledoux (1964) e Iwasaki et al. (1988). Sugere-se que este fato pode ter ocorrido devido ao tipo de processamento utilizado nas amostras ou também poderia indicar uma característica específica das espécies em questão. Jackowiak et al. (2009) analisando línguas de morcegos (Rousettus aegyptiacus) não encontraram sulcos medianos na superfície dorsal, freqüentemente presentes em outras espécies de morcegos. Assim, Jackowiak (2006) sugere que esta característica seja peculiar de cada espécie estudada.

Apenas na espécie Alouatta caraya foi observado, na superfície dorsal, uma proeminência lingual, estando localizada na metade do corpo até o início da base da língua. Sendo a mesma responsável por aumentar o atrito dos alimentos entre o palato superior e superfície dorsal da língua, desempenhando um importante papel durante a mastigação. Sua função é favorecida pelas robustas papilas linguais presentes nesta região (Kobayashi et al. 2003). Segundo Nonaka et al. (2008) a presença de uma proeminência lingual nesta região é considerada como uma característica estrutura de animais herbívoros. Sendo assim compatível com a dieta herbívora que estes primatas da espécie Alouatta caraya apresentam. Na proeminência lingual dos bugios-pretos fêmeas, foram visualizados apenas robustas e multifilamentosas papilas filiformes. No entanto, muitos autores descrevem a existência de papilas cônicas e lenticulares nesta região (Tadjalli \& Pazhoomand 2004, Shao et al. 2010).

Foram observadas papilas linguais apenas ao longo da superfície dorsal das línguas tanto das espécies Callithrix penicillata quanto da Alouatta caraya. Esta característica também foi relatada, por exemplo, em sapos por Iwasaki \& Kobayashi (1989), tartarugas por Lemell et al. (2000), ratos por Iwasaki et al. (1997), leões-marinhos por Yoshimura et al. (2002), botos por Guimarães (2006), preguiças-de-coleira por Benetti (2009), morcegos por Iwasaki et al (1986), Park et al. (2009) e Jackowiak et al. (2009), mico-leão-dourado por Burity et al. (2009), pandas por Pastor et al.(2008) e macaco-japonês por Emura et al. (2002) e Iwasaki et al. (1992), confirmando este padrão nas mais diferentes classes de animais.

Na superfície dorsal das línguas das duas espécies de primatas analisadas observou-se a presença de quatro tipos distintos de papilas linguais: filiforme, fungiforme, valada e folhada, apresentando assim uma distribuição semelhante à de outros mamíferos, principalmente com as demais espécies de primatas (Iwasaki et al. 1992, Emura et al. 2002, Burity et al. 2009). Entretanto indo ao contrário do relatado por Branco et al. (2012), os quais descreveram a existência de apenas três tipos de papilas línguas em animais adultos da espécie Callithrix penicillata.

As papilas filiformes observadas nos animais estavam distribuídas densamente no ápice e corpo da língua, estando dispostas ao redor das papilas fungiformes como já relatado por outros autores (Watanabe et al. 1992). A disposição entre as papilas fungiformes e a ausência de poros gustativos indica uma função mecânica de proteção da mucosa lingual pelas papilas filiformes (Emura et al. 2000, Jackowiak 2006). Já outros autores sugerem que essas mesmas papilas estão relacionadas ao processo de mastigação, uma vez que aumentam o atrito produzido pela língua durante o movimento e trituração dos alimentos, e também na função de limpeza corporal (Iwasaki 1992, Pastor et al. 2008, Lorych et al. 2009).

O pouco desenvolvimento e formatos variados das papilas filiformes nos animais filhotes das duas espécies analisadas está de acordo com o observado em recém-nascidos humanos (Ragia \& Nour 1998), macacos-japoneses filhotes (Emura et al. 2002) e morcegos recém-nascidos (Lorych et al. 2009). Segundo Faria (1989) nas duas semanas de vida os filhotes de alguns primatas começam a experimentar frutas maduras, mas sem deixar a amamentação, que finda aos dois meses, e assim sugerimos que o desenvolvimento das papilas filiformes tende a ocorrer de forma gradativa durante este período de mudança de dieta.

De maneira geral, a estrutura das papilas filiformes foi idêntica em toda superfície dorsal da língua nos animais jovens e adultos, corroborando com as descrições no Macaco-vervet (Cercopithecus aethiopus) (Emura et al. 2001). 0 formato e a orientação no sentido caudal observadas nestas papilas facilitam a aderência na superfície da língua, auxiliando assim o transporte dos alimentos sólidos e líquidos em direção à faringe (Ojima 2001). A ausência de papilas filiformes no animal adulto de Callithrix penicillata pode estar relacionada ao desgaste ocasionado pelo atrito constante de alimentos sólidos, como sementes e rações especificas, durante toda sua vida.

As estruturas das papilas fungiformes em Callithrix penicillata e Alouatta caraya filhotes foram menos desenvolvidas do que as papilas encontradas com formato arredondado e convexo (formato de "cogumelo") nos animais adultos. $\mathrm{O}$ formato de "cogumelo" das papilas fungiformes já se encontra bem estabelecido e descrito na literatura para diversas espécies de mamíferos adultos (Banks 1992, Kumar et al.1998, Samuelson 2007). Em humanos (Witt et al. 1997) foi observado que as papilas fungiformes apresentam uma forte tendência de crescimento individual. Tal fato pode justificar um maior desenvolvimento das papilas fungiformes encontrada nos animais mais velhos.

Sendo que tais papilas em ambas as espécies analisadas se encontravam distribuídas no ápice e corpo da língua. 
Esta característica também foi relatada em diversas outras espécies de primatas (Emura et al. 2002, Burity et al. 2009). A maior densidade destas papilas observadas no ápice da língua coincidem com relatos em animais frugívoros e insetívoros (Ciuccio et al. 2008, Jackowiak \& Godynicki 2005), que possuem hábitos alimentares similares aos do sagui-de-tufo-preto e em parte com os bugios-pretos. Resultados semelhantes também foram observados em outros primatas neotropicais como em Callithrix jacchus e Cebuella pygmaea (Kubota \& Hayama 1964). Na espécie Alouatta caraya a presença de um arranjo com cerca de três papilas fungiformes lateralmente (direita e esquerda) no corpo da língua, não foram relatados na literatura consultada. Podemos assim sugerir, que estes arranjos de papilas fungiformes, sejam particulares para a espécie Alouatta caraya.

A presença de poros gustativos observados na superfície das papilas fungiformes indica uma função gustativa nas mesmas (Pastor et al. 2008), podendo estar relacionadas proporcionalmente à variedade de alimentos que a espécie animal consome (Hwang \& Lee 2007). Tais poros também foram encontrados na superfície das mesmas papilas em macacos e humanos (Kobayashi et al. 2004). Diferentemente nas línguas dos bugios-pretos foram observadas poucas papilas fungiformes, provavelmente devido à dieta mais restrita que estes primatas apresentam. As alterações estruturais nas papilas fungiformes associadas à idade mais avançada observadas em Callithrix penicillata adulto, podem sugerir que ocorram perdas na capacidade gustativa nesses animais.

A presença de papilas valadas, observadas na região caudal da língua, corroboram com diversos relatos para outros mamíferos (Emura et al. 2004, Pastor et al. 2008). Em primatas o padrão de distribuição de tais papilas no formato de "V", tendo o ápice do "V" em direção a epiglote, parece estar bem estabelecido na literatura (Kobayashi et al. 2004). Contudo, a quantidade das mesmas varia conforme a espécie animal. Sendo que no macaco-esquilo (Iwasaki et al. 1988) observou-se uma única papila valada, no macaco-japonês e macaco-comedor-de-caranguejo haviam quatro papilas (Iwasaki et al. 1992, Kobayashi et al. 2004) e em humanos de cinco a doze papilas. Para os saguis-de-tufo-preto foi constatada a presença de 3 papilas valadas, estando de acordo assim com a descrição feita por Hershkovitz (1977), o qual descreve este valor para os calitriquídeos. E nas línguas dos bugios-pretos foram observadas um arranjo triangular de três papilas valadas, estando assim de acordo com as descrições para esta mesma espécie por Machida et al. (1967).

Morfologicamente as papilas valadas de Callithrix penicillata e Alouatta caraya filhotes possuíam tamanho menor do que as mesmas estruturais nos animais mais velhos, corroborando assim com os estudos em morcegos realizados por Lorych et al. (2009). Segundo Kubota (1988) esta diferença se deve aos hábitos alimentares do animal em determinada faixa etária, assim, o menor desenvolvimento das papilas valadas no animal filhote está relacionada a uma dieta mais líquida.

Segundo Pastor et al. (2008) os mamíferos apresentam duas morfologias distintas para as papilas valadas: simples ou composta. 0 tipo simples apresenta uma parte central rodeada por um sulco profundo. Já as compostas possuem uma área central dividida por sulcos secundários. Em Callithrix penicillata e Alouatta caraya as papilas valadas são do tipo simples, assim como ocorre em tigres (Emura et al. 2004), morcegos (Jackowiak et al. 2009) e micos-leões-dourados (Burity e al. 2009). Nestes sulcos localizam-se ductos provenientes de glândulas serosas, que por meio de sua secreção estão relacionados com a limpeza destas papilas e o aumento do paladar (König \& Liebich 2004).

Na superfície dorsal das papilas valadas estudadas dos saguis-de-tufo-preto foram observados poros gustativos, indicando assim uma função gustativa das mesmas. Resultados semelhantes também foram relatados em tatus (Ciuccio et al. 2008) e preguiças-de-coleira (Benetti 2009). No entanto, na superfície dorsal das papilas valadas dos bugios-pretos não foram observados poros gustativos, assim como em alguns caprinos (Kumar et al. 1998) e cervídeos (Zheng \& Kobayashi 2006).

A localização das papilas folhadas na região látero-lateral da língua nos animais analisados foram condizentes com diversas citações na literatura (Emura et al. 2002, Kobayashi et al. 2004, Yoshimura et al. 2009). Morfologicamente as papilas folhadas são caracterizadas por dobras paralelas com formato de folhas separadas entre si por uma invaginação da membrana mucosa (Banks 1992). Em nosso estudo os saguis-de-tufo-preto apresentaram papilas folhadas com 3 dobras, em contrapartida os bugios-pretos apresentaram 7- 8 dobras. Na literatura a razão da presença ou ausência das papilas folhadas em determinados animais ainda não está bem esclarecida. Shindo et al. (2006) relacionaram a presença de tais papilas com os métodos de mastigação e da dieta dos animais. E no caso de animais herbívoros tais autores sugerem que as papilas folhadas na parte posterior da língua apresentem uma importante função sensorial.

Segundo Mahmoud et al. (2002) tem-se uma relação entre a presença de papilas folhadas com o número de papilas valadas que um animal apresenta. Os autores observaram que a presença de papilas folhadas bem desenvolvidas em equinos, possuem relação com o pequeno número de papilas valadas nesta espécie. Já para a espécie bovina as papilas folhadas são pouco desenvolvidas, mas possuem grande quantidades de papilas valadas.

Ainda sobre tais estruturas, observou-se a presença de poros gustativos nos saguis-de-tufo-preto analisados, evento este também observado por Junqueira \& Carneiro (2005), indicando assim uma função gustativa para as mesmas. Contudo em Alouatta caraya não foram observados poros gustativos em suas superfícies. No entanto, os botões gustativos destas papilas podem estar presentes em seus sulcos profundos, como relatados em Equus asinus (Mahmoud et al. 2002).

A ausência de papilas linguais na raiz da língua, próxima à epiglote, com a presença de mucosas pregueadas nas duas espécies de primatas analisadas corroboram com relatos descritos em raposas (Iwasaki et al. 1988). Ainda nesta mesma região foram observados diversos forames de ductos das glândulas salivares em grande quantidade nos 
filhotes e jovens de saguis-de-tufo-preto e Alouatta caraya, ao contrário da pequena quantidade observada nos animais mais velhos.

\section{CONCLUSÕES}

Com o material analisado e a técnica empregada pôde-se constatar que as línguas dos saguis-de-tufo-preto (Callithrix penicillata) e dos bugios-pretos (Alouatta caraya) analisadas apresentaram papilas linguais similares às descritas para outras espécies de primatas. As línguas possuíam diferenças morfológicas, principalmente para alguns tipos de papilas linguais, em detrimento do avançar da idade dos animais.

De forma geral, nas espécies Callithrix penicillata e Alouatta caraya, foram observados quatro tipos de papilas linguais, sendo estas: filiforme, fungiforme, valada e folhada.

As línguas dos bugio-pretos apresentavam características de animais com dietas herbívoras. Possuindo desta forma papilas filiformes com formatos variados (coroa, lança, multifilamentar), de acordo com as regiões da língua, e também robustas papilas, principalmente na proeminência lingual. Já as línguas dos saguis-de-tufo-preto apresentaram características de animais onívoros. Com papilas filiformes em formato de coroa, grandes quantidades de papilas fungiformes e desenvolvidas papilas folhadas.

Observou-se que as modificações das papilas linguais que ocorrem nos animais após o nascimento apresentaram correlação com a mudança nos tipos de alimentos consumidos. Sendo presumível então concluir que as dietas possuem relação direta para com as alterações morfológicas e estruturais das línguas nos animais ora aqui analisados.

Agradecimentos.- Ao Programa de Iniciação Científica da Universidade de São Paulo pela concessão da bolsa de Iniciação Científica: PIBIC-SANTANDER. Ao criadouro Comercial de Animais Selvagens (AJBSoares), localizado em Atibaia/SP, e à Médica Veterinária Marta Brito Guimarães.

\section{REFERÊNCIAS}

Auricchio P. 1995. Primatas do Brasil. Terra Brasilis, São Paulo. 168p.

Banks W.J. 1992. Histologia Veterinária Aplicada. 2ª ed. Manole, São Paulo. 428p.

Benetti E.J., Pícoli L.C., Guimarães J.P., Motoyama A.A., Miglino M.A. \& Watanabe L.S. 2009. Characteristics of filiform, fungiform and vallate papillae and surface of interface epithelium-connective tissue of the maned sloth tongue mucosa (Bradypus torquatus, Iliger, 1811): Light and Scanning Electron Microscopy Study. Anat. Histol. Embryol. 38(1):42-8. doi: 10.1111/j.1439-0264.2008.00890.x

Branco E., Pereira W.L.A., De Lima A.R., Franciolli A.L.R., Rici R.E.G., Miglino M.A., Muniz J.A.P.C. \& Imbeloni A. 2012. Ultrastructural aspects of Callithrix penicillata lingual papillae. Microsc. Res. Tech. 75(3):282-4. doi: 10.1002/jemt.21055.

Burity C.H.F., Silva M.R., Souza A.M., Lancetta C.F.F., Medeiros M.F. \& Pissinatti A. 2009. Scanning electron microscopic study of the tongue in golden-headed lion tamarins, Leontopithecus chrysomelas (Callithrichidae: Primates). Revta Bras. Zool. 26:323-327.

Castro C.S.S. 2003. Tamanho da área de vida e padrão de uso do espaço em grupos de saguis Callithrix jacchus (Linnaeus) (primates callitrichidae). Rev. Bras. Zool. 20:91-96.

Ciuccio M., Estecondo S. \& Casanave E.B. 2008. Scanning electron microscopy study of the dorsal surface of the tongue in Zaedyus pichiy (Mammalia, Xenarthra, Dasypodidae). Int. J. Morph. 26:13-18.
Clark J.M. 1994. The common marmoset (Callithrix jacchus). Ann. News $7(2): 1-8$

Doran G.A. \& Baggett H A. 1971. Structural and functional classification of mammalian tongues. J. Mammal. 52:427-429.

Emura S., Tamada A., Hayakawa D., Chen H. \& Shoumura S. 2000. Morphology of the dorsal lingual papillae in the bush dog (Speothos venaticus). Okaj. Fol. Anat. 77:137-141.

Emura S., Hayakawa D., Chen H., Shoumura S., Atoji Y. \& Agungpriyono S. 2001. SEM study on the dorsal lingual surface of the lesser dog-faced fruit bat, Cynopterus brachyotis. Okaj. Fol. Anat. 78:123-128.

Emura S., Hayakawa D., Chen H. \& Shoumura S. 2002. Morphology of the dorsal lingual papillae in the Japanese Macaque and Savanna Monkey. Anat. Histol. Embriol. 31:313-316.

Emura S., Hayakawa D., Chen H. \& Shoumura S. 2004. Morphology of the lingual papillae in the tiger. Okaj. Fol. Anat. 81:39-44.

Faria D.S. 1989. 0 estudo de campo com mico estrela no Planalto Central Brasileiro. p.109-121. In: Ades C. (Ed.), Etologia de Animais de Homens. Edicon/dusp, São Paulo.

Guimaraes J.P. 2006. Análise morfológica da superfície de interface epitélio - tecido conjuntivo da face dorsal da língua de cetáceos (ordem Cetacea, subordem Odontoceti), empregando os métodos de microscopia de luz e eletrônica de varredura. Tese de Doutorado, Faculdade de Medicina Veterinária e Zootecnia, Universidade de São Paulo, São Paulo, SP. 94p.

Hershkovitz P. 1977. Living New World Monkeys (Platyrrhini) with an introduction to Primates. Vol.1. University of Chicago Press, Chicago. $1117 \mathrm{p}$.

Hwang H. \& Lee J. 2007. Morphological study on the dorsal lingual papillae of Myotis macrodactylus. Kor. J. Elect. Microsc. 37:147-156.

Iwasaki S., Miyata K., Wakasugi C. \& Kobayashi K. 1986. Scanning electron microscopic observations of the dorsal tongue surface in the Japanese house bat, Pipistrellus abramus. J. Mammal. Soc. Japan 11:155-164.

Iwasaki S., Miyata K. \& Kobayashi K. 1988. Scanning-electron-microscopic study of the dorsal lingual surface of the squirrel monkey. Acta Anat. 132:225-229.

Iwasaki S. \& Kobayashi K. 1989. Fine structure of the lingual dorsal epithelium in the bullfrog, Rana catesbeiana. Zool. Sci. 6:259-267.

Iwasaki S. 1992. Examination of the dorsal lingual surface of the Crab-eating Monkey Macaca irus by Scanning Electron Microscope. J. Mammal. Soc. Japan 17:49-57.

Iwasaki S., Yoshizawa H. \& Suzuki K. 1992. Fine structure of the dorsal lingual epithelium of the japanese monkey Macaca fuscata fuscata. Acta Anat. 144:267-277.

Iwasaki S., Yoshizawa H. \& Kawahara I. 1997. Study by scanning electron microscopy of the morphogenesis of three types of lingual papilla in the rat. Anat. Rec. 247:528-541.

Jackowiak H. \& Godynicki S. 2005. The distribution and structure of the lingual papillae on the tongue of the bank vole Clethrionomys glareolus. Fol. Morph. 64:326-333.

Jackowiak H. 2006. Scanning electron microscopy study of the lingual papillae in the European mole (Talpa europea, L., Talpidae). Anat. Histol. Embriol. 35:190-195.

Jackowiak H., Lorych T.J. \& Godynicki S. 2009. The microstructure of lingual papillae in the Egyptian fruit bat (Rousettus aegyptiacus) as observed by light microscopy and scanning electron microscopy. Arch. Histol. Cytol. 72:13-21.

Junqueira L.C. \& Carneiro J. 2005. Histologia básica. 10aㅡ ed. Guanabara Koogan, Rio de Janeiro. 524p.

Kobayashi K., Kumakura M., Yoshimura K., Nonaka K., Murayama T. \& Henneberg M. 2003. Comparative morphological study of the lingual papillae and their connective tissue cores of the koala. Ann. Embriol. 206:247-254.

Kobayashi K., Kumakura M., Yoshmura K., Takahashi M., Zeng J.H., Kageyama I., Kobayashi K. \& Hama N. 2004. Comparative morphological studies on the stereo structure of the lingual papillae of selected primates using scanning electron microscopy. Ann. Anat. 186:525-530. 
König H.E. \& Liebich H. J. 2004. Anatomia dos Animais Domésticos: texto e atlas colorido. Vol.2. Artmed, Porto Alegre, RS.

Kubota K. \& Hayama S. 1964. Comparative anatomical and neurohistological observations on the tongues of pigmy and common marmosets. Ann. Rec. 150:473-486.

Kubota K. 1988. Guide Book of Anatomy: the way to clarification of the mastication system. Nihon-Shika-Hyoron, Tokyo, p.50-65.

Kumar P., Kumar S. \& Singh Y. 1998. Tongue papillae in Goat: a scanning electron-microscopic study. Anat. Histol. Embriol. J. Vet. Med. 27(6):355357.

Ledoux L.J. 1964. A língua do sagui (Callithrix jacchus). F. Clin. Biol. 33:2341.

Lemell P., Beisser C.J. \& Weisgram J. 2000. Morphology and function of the feeding apparatus of Pelusios castaneus (Chelonia; Pleurodira). J. Morphol. 244:127-135.

Lorych T.J., Jackowiak H., Skieresz-Szewczyk K. \& Godynicki S. 2009. Morphology and morphometry of lingual papillae in adult and newborn Egyptian fruit bats (Rousettus aegyptiacus). Anat. Histol. Embriol. 38:370-376.

Machida H., Perkins E. \& Giacometti L. 1967. The anatomical and histochemical properties of the tongue of primates. Fol. Primat. 5(4):264279.

Mahmound M.M., Elnaeim A., Zayed A.E. \& Leiser R. 2002. Morphological characteristics of the tongue and its papillae in the donkey (Equus asinus): a light and scanning electron microscopical study. Ann Anat. 184(5):473-480.

Nonaka K., Zheng J.H. \& Kobayashi K. 2008. Comparative morphological study on the lingual papillae and their connective tissue cores in rabbits. Okaj. F. Anat. Jap. 85(2):57-66.

Nowak R.M. 1999. Walker's mammals of the world. Vol.2. $6^{\text {th }}$ ed. The Johns Hopkins University Press, Baltimore. 836p.

Ojima K. 2001. Functional role and angioarchitectural arrangement of the filiform papillae on the medio-dorsal surface of the beagle dog tongue. Ann. Anat. 183:325-329.

Park J. \& Lee J.H. 2009. Comparative morphology of the tongue of Miniopterus schreibersi fuliginosus and Pipistrellus savii. Kor. J. Elec. Microsc. 39(3):267-276.
Pastor J.F., Barbosa M. \& Paz F.J. 2008. Morphological study of the lingual papillae of the giant panda (Ailuropoda melanoleuca) by scanning electron microscopy. J. Anat. 212:99-105.

Ragia E. \& Nour E. 1998. Morphology and structure of filiform papillae of neonates: a light and scanning electron microscopic study. East Medit. Health J. 4:128-136.

Samuelson D.A. 2007. Tratado de Histologia Veterinária. Elsevier, São Paulo. $544 \mathrm{p}$.

Santini M.E.L. 1985. Alimentação e padrões de atividade de Alouatta caraya (Primates, Cebidae), reintroduzidos no Parque Nacional de Brasília. Tese de Mestrado, Universidade de Brasília, DF. 133p.

Shao B., Long R., Ding Y., Wang J.L., Ding L. \& Wang H. 2010. Morphological adaptations of yak (Bos grunniens) tongue to the foraging environment of the Qinghai-Tibetan Plateau. Am. Soc. Anim. Sci. 88(8):2594-2603.

Shindo J., Yoshimura K. \& Kobayashi K. 2006. Comparative morphological study on the stereo-structure of the lingual papillae and their connective tissue cores of the American Beaver (Castor canadensis). Okaj. F. Anat. Jap. 82(4):127-138.

Sonntag C.F. 1921. The Comparative Anatomy of the Tongues of the Mammalia. II. Family. 1. Simiidae. Proc. Zool. Soc. 1:1-29.

Tadjalli M. \& Pazhoomand R. 2004. Tongue papillae in lambs: a scanning electron microscopic study. Small Rumin. Res. 54:157-164.

Watanabe I., Inokuchi T., Yamada E. \& Semprini M. 1992. Distribution of the taste buds on the lingual mucosa of the Macaca fuscata: scanning electron microscopy study. Revta Bras. Ciênc. Morfol. 9:7-13.

Witt M. \& Reutter K. 1997. Scanning electron microscopical studies of developing gustatory papillae in humans. Chem. Sen. 22(6):601-612.

Yoshimura K., Shindoh J. \& Kobayashi K. 2002. Scanning electron microscopy study of the tongue and lingual papillae of the California Sea Lion (Zalophus californianus californianus). Anat. Rec. 267:146-153.

Yoshimura K., Hama N., Shindo J., Kobayashi K. \& Ikuo Kageyama I. 2009. Light and scanning electron microscopic study on the tongue and lingual papillae of the common Hippopotamus, Hippopotamus amphibius amphibius. Anat. Rec. 292:921-934.

Zheng J. \& Kobayashi K. 2002. Comparative morphological study on the lingual papillae and their connective tissue cores (CTC) in Reeves' muntjac deer (Muntiacus reevesi). Ann. Anat. 188:555-564. 